\title{
tic\&société
}

Vol. 3, $n^{\circ}$ 1-2| 2009

TIC et diasporas

\section{Les sites web diasporiques : un nouveau genre médiatique?}

\section{Claire SCOPSI}

\section{(2) OpenEdition \\ Journals}

Édition électronique

URL : http://journals.openedition.org/ticetsociete/640

DOI : 10.4000/ticetsociete. 640

Éditeur

Association ARTIC

\section{Référence électronique}

Claire SCOPSI, «Les sites web diasporiques : un nouveau genre médiatique ? », tic\&société [En ligne], Vol. 3, n 1-2 | 2009, mis en ligne le 20 avril 2019, consulté le 04 mai 2019. URL : http:// journals.openedition.org/ticetsociete/640; DOI : 10.4000/ticetsociete.640 
tic\&société - 3 (1-2), 2009

\title{
Les sites web diasporiques: un nouveau genre médiatique?
}

\author{
Claire Scopsi \\ DICEN Dispositifs d'information et de communication à l'ère du numérique \\ Conservatoire national des Arts et métiers \\ 2, rue Conté \\ 75003 PARIS \\ claire.scopsi@cnam.fr
}

Claire Scopsi a assuré les fonctions de chef de projet informatique documentaire et multimedia dans diverses entreprises du secteur des services et des médias de 1993 à 2007. Docteur en sciences de l'information et de la communication, elle est maître de conférences au Conservatoire national des Arts et métiers (CNAM) et assure des enseignements en techniques documentaires et méthodologie de projet à l'Institut national des Techniques de la documentation (INTD). Ses travaux d'ethnométhodologie au sein du laboratoire DICEN (Dispositifs d'information et de communication en environnement numérique) du CNAM portent sur les dispositifs d'accès aux réseaux numériques (téléboutiques, cybercafés) des quartiers ethniques et sur la production médiatique des communautés transnationales connectées. 


\title{
Les sites web diasporiques: un nouveau genre médiatique?
}

\begin{abstract}
Résumé
Depuis le début des années 2000, les TIC (téléphone mobile, mail ou web) jouent un rôle croissant dans le quotidien des migrants. Dans la continuité des journaux de migrants, les sites web rédigés par des migrants se donnent pour mission de relier les membres de communautés qui de plus en plus se désignent elles-mêmes comme des diasporas. Cet article s'interroge sur la définition et la méthode d'observation de ces "sites web diasporiques" et conclut à leur possible contribution à l'élaboration ou au renforcement du sentiment d'appartenance communautaire au sein des populations dispersées.
\end{abstract}

Mots-clés: Diaspora, communication des migrants, web diasporique, identité diasporique, migrant connecté.

\begin{abstract}
Since the beginning of the millenium, ICTs (cell phones, mail or web sites) have come to play an increasing part in the life of migrants. Like migrant newspapers before them, web sites are created by migrants in order to maintain a link between the members of communities who increasingly refer to themselves as "diaspora ». This paper questions the definition of and methods for observing these " diasporic web sites " and concludes that they may help develop and strengthen the feeling of a common identity among dispersed communities.
\end{abstract}

Keywords: Diaspora, diasporic web site, diasporic identity, connected migrant.

\section{Resumen}

Desde principios de los años 2000, las TIC (telefonía móvil, correo electrónico o web) desempeñan un papel cada vez más importante en el día a día de los emigrantes. En la continuidad de los diarios de los emigrantes, los sitios web redactados por emigrantes tienen como misión unir los miembros de las comunidades, que se caracterizan, cada vez más, como diásporas. Este artículo estudia la definición y el método de observación de dichos sitios web de la diáspora. Se concluye sobre la contribución a la 
Les sites web diasporiques : un nouveau genre médiatique?

elaboración, o al refuerzo del sentimiento de pertenencia comunitaria en el seno de las poblaciones dispersas.

Palabras clave: Diáspora, comunicación de los emigrantes, web de la diáspora, identidad de la diáspora, emigrante conectado. 


\section{Claire SCOPSI}

\section{Introduction}

Les technologies de communication comptent parmi les plus récentes innovations qui ont modifié les conditions de vie des migrants, leur perception de l'espace et leur relation avec la nation mère. Pour beaucoup d'entre eux, le développement des moyens de transport, l'abaissement des coûts et de la durée des voyages ont autorisé le va-et-vient, cette succession de présences et d'absences qui conduit à une double présence pour ainsi dire alternée. Dès les années 1970, les travailleurs émigrés intervenaient ponctuellement dans la vie du village ou de la famille "au pays" depuis le téléphone du foyer de travailleurs (Gonin, 2001). Puis, les peuples dispersés se sont rassemblés autour des mêmes feuilletons ou des retransmissions des grands événements publics du "homeland " diffusés par les canaux satellitaires (Dayan et Katz, 1996).

Les technologies numériques s'inscrivent donc bien dans une continuité, celle de l'intensification des communications : d'un unique micro connecté à internet, les migrants les mieux équipés communiquent par visioconférence, échangent films et photos, écoutent la musique et les informations des webmédia locaux tandis que les moins favorisés envoient de l'argent et des mails qui seront réceptionnés via les cybercafés. Les téléphones cellulaires vendus ou donnés par les migrants dans leur pays d'origine se sont rapidement répandus jusque dans les zones rurales les plus reculées (Chéneau-Loquay, 2004), et leur permettent de rester connectés, joignables de partout et à tout moment. Seule la conjonction d'une extrême pauvreté et d'interdictions politiques draconiennes peut aujourd'hui contraindre un individu à un exil radical. À la " double absence » nostalgique décrite par Abdelmalek Sayad (1991), s'est peu à peu substituée la "double présence du migrant connecté " de Dana Diminescu (2005) qui nous conduit à une nouvelle lecture de l'état de migrant.

Comme l'avait déjà souligné Sayad (1985) en montrant la complémentarité des messages écrits ou oraux confiés à des intermédiaires, postés ou enregistrés sur des cassettes de magnétophones dans les familles de migrants, les usages des technologies de la communication sont combinés et l'on observe des continuités, des juxtapositions et des ruptures entre nouveaux médias et outils traditionnels. Les migrants combinent téléphonie fixe et mobile selon l'objectif, le coût, la commodité et la carte téléphonique n'exclut pas l'abonnement, ni le mail le courrier. Seule la disparition progressive de certains supports et équipements (cassettes et lecteurs) conduit à l'abandon de pratiques antérieures. 
Les sites web diasporiques : un nouveau genre médiatique?

Le téléphone portable a été décrit par Dana Diminescu (2002) dans les communautés de migrants roumains de la fin des années 1990 comme un outil d'« installation relationnelle », c'est à dire comme le dispositif par lequel le migrant organise sa vie en mobilité. Outil d'intégration professionnelle dans le " hostland ", il permet d'être joint pour les "boulots occasionnels ou informels qui sont la première étape dans le parcours des nouveaux arrivants ». II est aussi le vecteur de la double présence comme l'était vingt ans auparavant le téléphone fixe installé dans les foyers pour les travailleurs immigrés maliens (Gonin, 2001). Il permet d'intervenir à distance dans la vie quotidienne du village d'origine, de contrôler les travaux de construction d'une maison ou de suivre les naissances dans les troupeaux. À cet égard le téléphone est le medium de la participation de l'individu à ses communautés.

Le mail, par son faible coût et sa rapidité, s'est imposé naturellement comme le remplaçant des lettres de migrants. La visioconférence en revanche trouve plus difficilement sa place. En 2001, la première tentative de deux cybercafés associés, le Métissacana à Dakar et le Vis@Vis à Paris, pour mettre en relation les émigrés africains et leurs familles par visiophonie publique s'est soldée par un échec. Puis, le procédé s'est propagé peu à peu et il n'est plus exceptionnel en 2009 d'en observer l'usage dans les cybercafés fréquentés par les migrants. Mais, malgré quelques similitudes, on ne peut pas dire que les webcams remplacent exactement les cassettes vidéo que s'envoyaient les familles car l'image ainsi produite est volatile et ne peut être revisionnée ni être conservée en souvenir. En outre, elle nécessite une ligne à haut débit et une présence simultanée délicate à organiser depuis des cybercafés situés dans des pays soumis au décalage horaire. La visioconférence est donc plutôt (mais massivement) utilisée par les migrants aisés équipés à domicile.

Dans ce contexte d'usages, cet article a pour propos d'esquisser la méthodologie de l'étude "manuelle », c'est-à-dire sans outils de traitement automatique, d'une production médiatique que nous appellerons « web diasporique " en postulant qu'elle joue un rôle actif dans l'émergence ou le renforcement du sentiment identitaire dans les communautés dispersées. L'étude repose sur l'observation entre 2005 et 2009 d'une trentaine de sites web réalisés par des migrants isolés ou des associations de migrants d'origines diverses ${ }^{1}$. Après un bref état du contexte théorique des recherches sur les diasporas et leur présence sur le net (partie 1), nous évoquerons les limites méthodologiques rencontrées pour l'identification, la conservation et l'exploitation de ces sites et proposerons une définition permettant d'isoler le web diasporique parmi les sites web de migrants (partie 2). Puis, nous présenterons les caractéristiques communes à ces sites qui permettent, au-delà

\footnotetext{
${ }^{1}$ La liste complète de ces sites figure à la fin de cet article.
} 
des spécificités culturelles, de les considérer comme un genre à part entière (partie 3).

\section{Des diasporas traditionnelles aux « digital diasporas " : repères théoriques}

Si la mondialisation est l'âge du risque, des mobilités et de l'information, alors les migrants connectés en sont l'archétype et ont beaucoup à nous apprendre sur nos sociétés modernes.

En effet, les technologies de l'information et de la communication (TIC) et les migrations tissent d'incessantes relations dont l'analyse doit dépasser la seule phénoménologie. Les pratiques informationnelles des migrants ne sont plus des comportements enfouis dans la sphère privée et spécifiques à une communauté et à une époque. Elles ont acquis une force et une dynamique durables et sont observables dans l'espace public.

Depuis 2000, une multitude de boutiques de téléphonie internationale, de cybercafés, de commerces de téléphones cellulaires créés par des migrants est apparue dans les quartiers ou les banlieues des métropoles occidentales où vivent des populations pluriethniques. Leur densité est exceptionnelle dans ces lieux traditionnellement voués au commerce ethnique, à l'instar du quartier de Château rouge dans le $18^{\mathrm{e}}$ arrondissement de Paris où l'on compte en 2009 plus de cent commerces de ce type, créés entre 2001 et 2003, répartis dans seulement une dizaine de rues. Tant et si bien que les grands opérateurs de télécommunications et les acteurs du transfert d'argent y ont peu à peu ouvert leurs franchises, profitant de cette attractivité (Scopsi, 2004 et 2008). Insensiblement, ces commerces technologiques " high tech » (ils ont exploité très tôt la voix sur IP ou la visioconférence) à la communication volontairement banalisée et ethnicisée se sont imposés comme les marqueurs urbains des quartiers de migrants.

Dans le même temps, des migrants, souvent les informaticiens de la diaspora, se sont appuyés sur les infrastructures de leur pays d'accueil pour ouvrir des sites web, des forums ou des portails d'information consacrés à leur pays et en assurer la présence sur la toile mondiale alors même que les conditions de création et d'exploitation de ces sites n'étaient pas encore réunies dans leur pays d'origine.

En 2002, Emmanuel Ma Mung s'étonne de compter un nombre croissant de références du terme diaspora dans les résultats du moteur de recherche Google et s'interroge sur l'acception, inattendue pour un chercheur en sciences sociales 
Les sites web diasporiques : un nouveau genre médiatique?

français, que prend ce mot dans les productions numériques des AfroAméricains. Le début des années 2000 semble en effet marquer un tournant dans l'appropriation par le grand public d'un vocable jusque là réservé à la seule communauté juive, puis, par analogie, à d'autres communautés dispersées (Arméniens, Grecs, Chinois), sans que les chercheurs aient pu se mettre pleinement d'accord sur une exacte définition. Parallèlement à un important travail de théorie de la diaspora (Cohen, 1999 ; Dufoix, 2003 ; Bruneau, 2004 ; Anteby-Yemini et al., 2005), un nombre croissant de travaux consacrés aux pratiques de communautés diverses (Philippins, Irlandais, Tamouls, Berbères, Chinois, Burundais et bien sûr la "black diaspora ") témoigne de l'intérêt des chercheurs internationaux pour l'étude de l'usage de l'internet par ces communautés. La revendication, bien visible sur l'internet, d'une identité diasporique par des communautés occidentales d'expatriés (Bretons, Basques) ou dans des contextes de trauma collectif réactivant d'anciennes blessures identitaires (catastrophe du cyclone Katrina) montre également que la construction d'une identité collective n'est pas limitée à un contexte postcolonial et peut émerger dans toute situation de dispersion.

Le portail Google Scholar permet de tracer l'apparition du terme « digital diaspora » dans des travaux à partir de 1997. Ce terme qui ne désigne d'ailleurs pas toujours des communautés ethniques mais par exemple des communautés de joueurs en réseau, est de plus en plus souvent repris depuis 2005 par la communauté scientifique internationale pour désigner les migrants connectés à internet. II n'a cependant pas encore trouvé d'équivalent en français.

La plupart de ces travaux rendent compte des pratiques du web, du mail, des réseaux sociaux ou du téléphone mobile par les populations étudiées et soulignent par le terme diaspora le caractère dispersé de la population émigrée (cas d'une population migrant et s'installant en plus d'un point hors du homeland) sans toutefois interroger l'apport théorique des TIC au concept de diaspora. Quelques travaux posent cependant la question du rôle joué par l'internet dans la mémoire d'un trauma collectif (Barrier, 2006), la représentation du territoire originel ou le processus de construction de l'identité diasporique dans le contexte postcolonial (Ignacio, 2006).

«I argue that Internet research can add to our understanding of postcoloniality (and postmodernity) in that it allows us to see the process of redefinition among self defined members of diasporas in a decentered space. Because of this, there is the 


\section{Claire SCOPSI}

potential for more voices to be heard simultaneously ${ }^{2}$ » (Ignacio, 2006, p.186).

Ces derniers travaux posent l'hypothèse d'un internet jouant non seulement un rôle de support des échanges et de faciliteur des relations entre membres d'une diaspora préexistante, mais aussi capable de favoriser et même d'activer le processus d'élaboration identitaire qui conduit à l'existence d'une diaspora. Pour faire écho à l'argumentation d'Emily Noelle Ignacio et tenter d'apporter une explication à l'accroissement parallèle du nombre de sites web et d'occurrences du terme diaspora sur le web, on peut avancer que si l'internet permet aux chercheurs d'entendre simultanément un nombre grandissant de voix diasporiques et d'en étudier les traces et les écrits, ces voix sont aussi entendues par les autres migrants car les écrits du web leur sont accessibles. En se propageant dans les populations d'immigrés les proclamations des sites web diasporiques suscitent des prises de consciences identitaires et le désir de se redéfinir comme communauté dispersée.

C'est pourquoi élaborer une méthode d'analyse des sites web de migrants, permettant d'évaluer leur contribution à l'identité diasporique revêt un intérêt pour la compréhension des migrations contemporaines.

\section{Collecte et définition des sites web diasporiques}

Mais l'étude des productions des diasporas sur internet présente un certain nombre de difficultés :

- Une difficulté liée à la conservation et l'exploitation des écrits du réseau, volumineux, mouvants et pour la plupart encore non archivés (Diminescu, 2005). Le projet Migr'archive ${ }^{3}$ de constitution d'une mémoire électronique pour la recherche sur les migrations apporte des pistes pour l'identification et l'archivage automatisées des traces numériques laissées par les migrants.

- Une double difficulté majeure à définir l'objet web diasporique. En effet, il s'agit d'extraire d'un ensemble encore majoritairement indifférencié - «les sites web» (comme on pourrait dire «les

\footnotetext{
2 « J'affirme que les travaux de recherche sur l'internet peuvent contribuer à notre compréhension de la postcolonialité (et de la postmodernité) en ce qu'ils nous permettent de voir le processus de redéfinition au sein des membres auto proclamés de diasporas dans un espace décentré. Pour cette raison, la possibilité est offerte à davantage de voix de se faire entendre simultanément. »

${ }^{3}$ Projet mené par le Groupe d'études sur l'usage des TIC dans les migrations (MSH Paris-Raspail) et l'Institut national de l'audiovisuel, autour des outils du dépôt légal du web. http://www.ticm.mshparis.fr/spip.php?article20 (accédé le $1^{\mathrm{er}}$ février 2009).
} 
Les sites web diasporiques : un nouveau genre médiatique?

livres »)-, un sous ensemble présentant suffisamment de caractéristiques communes pour constituer un genre que l'on appellera web diasporique. Or, la notion de diaspora est elle-même malaisée à définir et souvent controversée. Au minimum, on peut la définir comme une communauté dispersée, reliée par un imaginaire commun manifesté dans des productions culturelles ou médiatiques (Anderson, 1996). Mais comment alors éviter la tautologie qui consiste à définir le web diasporique comme un site alimenté par une diaspora, elle-même définie comme une communauté reliée par un site web?

- Enfin une difficulté épistémologique : faut-il privilégier la notion de genre, aborder donc les sites par leur structure et leur contenu de façon transversale, sans prendre en compte l'origine culturelle des auteurs ou respecter la posture anthropologique traditionnelle et s'intéresser aux sites d'une communauté particulière en tant que documents sur lesquels appuyer une étude culturellement centrée ?

Le travail d'analyse des sites web de migrants était lié à son début à l'étude d'un quartier très pluriethnique dans lequel l'identité affichée, revendiquée et simulée est un jeu marchand constant et ne se prête pas à une approche traditionnelle par ethnie. Aucune communauté n'a donc été privilégiée. Cette approche transversale présente de nombreuses limites car il n'est possible de maîtriser ni les langues, ni les contextes politiques et culturels des diverses communautés. Pour cette raison, l'étude des contenus ne peut que rester superficielle. En revanche, cette approche s'avère fructueuse pour une contribution à la théorie des diasporas car la juxtaposition des sites montre, au delà des spécificités culturelles, des récurrences dans les structures et les propos.

La première phase de la collecte s'est déroulée en 2005 et 2006 . Elle a succédé à un travail d'observation des cybercafés de migrants dans un quartier parisien. Les traces de connexion des navigateurs montraient l'intérêt des clients pour les sites consacrés à leurs pays d'origine, le travail s'est donc poursuivi sur le web. Près de quatre-vingts sites ont été identifiés et une cinquantaine ont été retenus comme diasporiques, soit parce qu'ils se présentaient explicitement comme tels en utilisant le terme diaspora, soit parce qu'ils se donnaient pour mission la mise en relation des membres dispersés de leur communauté. Ils ont été suivis pendant deux ans.

La collecte s'est effectuée en anglais et français dans le moteur de recherche Google ce qui a pour inconvénient d'éliminer un grand nombre de sites, notamment ceux qui utilisent un jeu de caractères non latins. Les sites suivis sont donc ceux qui sont rédigés ou qui comportent une version en français ou 


\section{Claire SCOPSI}

en anglais. Le volume parfois très important de pages ou d'échanges dans les forums n'a permis qu'un suivi global. L'étude s'est donc intéressée à la structure des sites, leurs rubriques, leurs pages de présentation, les grandes thématiques des forums, mais ne prend en compte qu'exceptionnellement les contenus.

Les sites ont de nouveau été consultés fin 2008. Plus de la moitié restaient vivants ${ }^{4}$ comme en témoignent les messages des forums ou les thèmes des billets, ce qui peut paraître surprenant car certains étaient créés par des personnes privées. La longévité, malgré le manque de moyens, est aussi une caractéristique de ces sites.

Une étape de définition s'avère indispensable car certains sites comportent le mot diaspora dans leur titre ou leur nom de domaine sans que rien dans leur fonctionnement ne le justifie. C'est le cas par exemple du site La vie en diaspora ${ }^{5}$, créé aux États-Unis en 2000 par des journalistes et resté depuis peu actif, qui s'adresse aux francophones émigrés aux États-Unis. Peut-on parler d'une diaspora francophone?

Cela nous oblige à revenir sur les travaux récents de théorie de la diaspora : absent du vocabulaire scientifique avant les années 1980 (hormis lorsqu'il désigne la diaspora juive) le terme diaspora est utilisé de façon hétérogène et controversée à la fin du $X X^{e}$ siècle et désigne des communautés extrêmement diverses (communauté juive, arménienne, grecque, chinoise, libanaise, tzigane, caribéenne ou afro-américaine) (Dufoix, 2003). Les pôles structurants sont de trois types: l'entrepreneuriat (communautés chinoises, libanaises), le religieux (communautés juives, grecques), le politique (Palestiniens, Arméniens).

Mais il ne désigne jamais, dans la langue courante, «la dispersion des Européens fondant leurs empires coloniaux à travers le monde ni non plus les migrations des travailleurs pauvres du Sud vers les pays européens riches... » (Schnapper, 2005, p.22). II est donc important de fixer le concept de diaspora pour une utilisation scientifique et le distinguer des emplois courants vides de sens.

Gabriel Scheffer (1993) propose trois caractéristiques essentielles: la conscience et le fait de revendiquer une identité ethnique ou nationale, l'existence d'une organisation politique, religieuse ou culturelle (une vie associative riche par exemple), l'existence de contacts réels ou imaginaires avec le territoire d'origine (éventuellement sous forme de mythe de retour).

\footnotetext{
${ }^{4}$ Mais ces sites avaient parfois changé de technologie, passant du html statique à une solution dynamique.

${ }^{5} \mathrm{http}: / /$ www.vieendiaspora.net/diaspora.htm (accédé le 20 janvier 2009).
} 
Les sites web diasporiques : un nouveau genre médiatique?

Il est à noter que, bien que le caractère construit voire imaginé et donc susceptible de subir des variations d'intensité de la diaspora soit reconnu (Anderson, 1996), la pérennité reste un important critère d'identification. « Pour que le concept de diaspora ait un sens précis et heuristique fécond, il faut éviter qu'il ne s'applique à toute forme de dispersion, provisoire, instable ou précaire » (Bruneau, 2004, p.20).

Mais ce dernier critère ne permet d'envisager la diaspora que comme entité durable constituée de longue date et non comme un processus évolutif. Cependant Alain Medam (1993) insiste sur le degré d'homogénéité et le dynamisme de l'organisation diasporique et distingue les diasporas cristallisées, durcies, stabilisées, de la fin du $\mathrm{XIX}^{\mathrm{e}}$ siècle, des diasporas fluides, mouvantes, flottantes, contemporaines et "en voie de constitution », ce qui permet de considérer aussi les étapes d'élaboration d'une diaspora. Nous avons pris en compte cette approche dans la sélection des sites et n'avons donc pas limité le choix aux grandes et pérennes diasporas.

À la lueur de ces définitions, procédons par élimination pour distinguer les sites web de diasporas:

II s'agit de sites témoignant de la conscience que les diasporas ont d'elles mêmes et porteurs de revendications identitaires; ils émanent obligatoirement des membres de la communauté. Cette exigence nous conduit vers un autre type de sélection que celle publiée en 2005 par Françoise Rollan qui concerne les sites utiles à l'étude des diasporas et mêle indistinctement les sites d'experts et les sites d'associations communautaires.

Nous éliminons donc de notre sélection :

- Les sites "sur les diasporas ॥ réalisés par des chercheurs ou des experts, et apportant des informations théoriques, historiques ou géopolitiques sur la notion de diaspora ou sur une diaspora particulière. Citons pour exemple le site des Irish diaspora studies de l'université de Bradford ${ }^{6}$ ou le site des Chinois de France animé par Pierre Picquart?.

- Les sites « vers les diasporas » émanant des gouvernements ou de représentants des gouvernements et s'adressant aux expatriés d'une nation pour les informer sur leurs droits et les avantages qui leur sont proposés dans le cadre notamment de leurs investissements. Ces sites ont fleuri à partir de 2000 lorsque les pays sources d'émigration ont perçu l'opportunité que représentait l'internet pour entretenir un

\footnotetext{
${ }^{6} \mathrm{http}: / /$ www.bradford.ac.uk/acad/diaspora/ (accédé le 20 janvier 2009).

7 http://www.chinoisdefrance.com/ (accédé le 20 janvier 2009).
} 


\section{Claire SCOPSI}

lien durable avec leur diaspora, susciter les investissements de leurs ressortissants de l'étranger ou maintenir les relations avec leurs « cerveaux " expatriés. Dans cette catégorie de sites le plus souvent signés par un ministère, citons marocains du monde du ministère chargé de la Communauté marocaine résidant à l'étranger ${ }^{8}$ ou the indian diaspora du High Level Committee on Indian Diaspora ${ }^{9}$.

Pour réduire le plus possible la sélection aux sites réellement écrits par les migrants, le repérage des marqueurs linguistiques de la migration est très utile. Dans chaque communauté il existe des mots par lesquels les migrants se désignent eux- mêmes ( viet-kieu » au Vietnam, " $\mathrm{RME}^{10}$ " au Maroc, les « jeviens » aux Comores...) et d'autres par lesquels ils désignent ceux qui restent dans le homeland ( blédards », " je-reste »). L'association de ces termes avec des locutions comme « notre village », « notre communauté », ou « diaspora » permet de réduire le bruit lors de la sélection.

La publication par les membres d'une communauté transnationale n'est cependant pas un critère suffisant car on ne peut considérer comme un élément suffisant l'existence de moyens de communication au sein d'une telle communauté sous peine de devoir considérer que tout site émanant d'une communauté dispersée suffit à l'élire comme diasporique. Les critères de cohésion de revendication identitaire nous aideront à sortir de ce cercle vicieux.

Ce critère nous conduit à éliminer des sites nostalgiques, rassemblant des émigrés autour de l'évocation du pays quitté sans déboucher sur une autre mission que cette évocation. Le site $\mathrm{ES}^{\prime} M \mathrm{MA}^{11}$ où les anciens habitants des quartiers centre d'Alger, le plus souvent des pieds noirs rapatriés, rassemblent anecdotes, souvenirs et photos de classe en est un exemple : l'évocation du territoire quitté ne conduit à aucune prise de position politique ou identitaire et le passé y est nettement dissocié du présent.

Un autre phénomène doit être pris en compte : dans nombre de pays du Sud (en Afrique notamment), où les infrastructures techniques sont insuffisantes et l'offre d'hébergement de sites inexistante, les portails nationaux se sont créés dans la diaspora et la publicité présente dans les pages s'adresse le plus souvent aux émigrés plus aisés que les habitants du homeland. Cela n'implique cependant pas que les contenus reflètent les préoccupations de la diaspora et ses modes de vie dans les pays de dispersion. Dans le cas du portail sénégalais Senewe $b^{12}$ par exemple, les contenus sont ceux d'un site d'actualité nationale.

\footnotetext{
${ }^{8}$ http://www.marocainsdumonde.gov.ma/ (accédé le 20 janvier 2009).

9 http://indiandiaspora.nic.in/ (accédé le 20 janvier 2009).

10 Ressortissants marocains de l'étranger.

${ }^{11} \mathrm{http}: / /$ esmma.free.fr/ (accédé le 20 janvier 2009).

12 http://www.seneweb.com/ (accédé le 20 janvier 2009).
} 
Les sites web diasporiques : un nouveau genre médiatique?

C'est pourquoi il faut, pour que la notion de site diasporique prenne sens, que le contenu du site serve le processus diasporique, c'est-à-dire qu'il entretienne la conscience ou la prise de conscience par les membres d'une identité commune, la proclame aux yeux du monde, fonde et organise les missions que la communauté s'assigne. Ces missions justifient à elles seules l'étude des diasporas car, manifestation même de la dynamique de la communauté transnationale, elles démontrent la puissance du processus diasporique susceptible à partir d'une perception imaginaire de quitter le monde virtuel pour produire de la valeur ou de l'influence politique.

Nous posons donc ici comme définition que les sites web diasporiques sont des sites produits par des communautés transnationales depuis un des lieux de dispersion, s'organisant autour d'un ou de plusieurs éléments culturels partagés (langue, religion, appartenance ethnique), s'adressant explicitement aux membres de la communauté dispersés dans le monde par la migration et éventuellement à la population restée dans le homeland en contribuant à la conscience du lien identitaire, à son affirmation publique et à sa concrétisation par des actions de revendication, de représentation ou de développement économique et culturel au profit de ses membres.

\section{Caractéristiques et spécificités des sites web diasporiques}

Le web au sens de données codées en html (statiques ou dynamiques) est partiellement le successeur de la presse communautaire. On retrouve notamment de nombreuses similitudes entre les rubriques et thématiques des journaux qualifiés par Jean-Paul Marthoz (2001) de dispositif de « va-et-vient communicationnel des diasporas " et celles des sites web du début des années 2000 :

- informations sur l'actualité du pays d'origine et du (ou des) pays d'accueil,

- prise de position dans la lutte contre les discriminations dont les membres de la communauté sont victimes,

- mise en valeur des réussites de la communauté, promotion de modèles positifs au sein du groupe contrastant avec le traitement pessimiste des médias dominants des pays d'accueil,

- informations et conseils administratifs concernant la naturalisation, les investissements professionnels ou les achats d'immobilier au pays, 
- publicités pour des produits de télécommunication, des agences de voyages, des sociétés de fret...

Mais le web permet plus que la presse écrite et certaines de ses caractéristiques intrinsèques influencent les contenus des sites de migrants pour leur conférer une identité propre. C'est le cas du caractère privé/public des contenus. L'une des plus surprenantes caractéristiques du web est en effet sa capacité à donner l'impression d'un entre-soi tout en étant potentiellement accessible par la totalité des individus connectés de la planète. Les sites de migrants affichent cette ambivalence et tiennent compte du fait qu'ils peuvent être lus à la fois par des internautes intégrés ou étrangers à la communauté. Une distinction éditoriale s'opère souvent entre les zones de publication officielles du site (pages html statiques ou contenus gérés automatiquement) et les forums plus informels. Les zones de publication prennent en charge l'information de la diaspora en relayant des fils RSS d'actualité concernant le homeland ou des informations sélectionnées par le ou les auteurs du site où l'on retrouve les thèmes habituels des médias diasporiques traditionnels. C'est aussi dans ces zones que les auteurs assument leur rôle de passeur culturel en s'adressant cette fois au monde entier. Les rubriques retraçant l'histoire et les chiffres clés de l'économie du pays d'origine, la liste minutieuse des lieux remarquables agrémentée des photos envoyées par les internautes, les recettes de cuisine traditionnelles concourent à la visibilité « urbi et orbi » de la culture du homeland tout autant qu'à entretenir les repères culturels des émigrés de deuxième et troisième générations nés à l'étranger. Le dictionnaire phonétique franco-arménien et les étonnantes "historiettes d'une mamie pas comme les autres" de Netarmenie.com, le savoureux lexique franco-ivoirien de nouchi.com, les traductions françaises de prénoms marocains de yabiladi.com témoignent de cette volonté de se représenter sur le web.

Le forum en revanche est le lieu du débat et de l'introspection identitaire. II est parfois le seul lieu où le lien, l'exil et l'identité sont clairement évoqués dans certains sites qui s'affichent simplement comme des sites d'information sur un pays. D'un accès plus limité, puisqu'il faut le plus souvent déclarer son pseudonyme même si aucune condition d'entrée n'est exigée, le forum rassemble essentiellement les membres de la diaspora. Le langage, plus relâché, y mêle les langues et l'argot de migrant :

« How do other people view U.S vietkieus? They must have a negative view right? When I came back to Saigon last year, I heard some people said that vietkieu from the U.S are very la'o $\mathrm{ca}^{13}$. "

\footnotetext{
${ }^{13}$ Forum http://asiafinest.com/ fil de discussion « U.S Vietkieus VS Others? », 22 janvier 2006.
} 
Les sites web diasporiques : un nouveau genre médiatique?

Les fils de discussion qui comptent le plus de "post " sont ceux consacrés à la migration et aux négociations avec les racines et la culture du hostland qui sont l'essence même de la migration. Cette négociation se cristallise sur des objets différents selon les cultures: le débat sur le mariage mixte occupe pendant plus d'un an la communauté de orientalement.com, celui, plus "libéré ", intitulé «les femmes maghrébines qui couchent avec des Occidentaux " génère plus de six cents posts en deux mois sur ibled.com. La reconnaissance de la langue arménienne et du génocide sont des thèmes importants des forums arméniens. Les blagues, les dessins humoristiques, l'autodérision sont présents dans la plupart des forums quelque soit l'origine culturelle :

«Quand tu fais quelque chose à Paris, quelqu'un d'autre à Paris le lendemain te dit ce que tu as fait, mais parce qu'il l'a appris de quelqu'un qui est aux Comores, qui a parlé la veille à quelqu'un qui était à Marseille, qui avait téléphoné à quelqu'un qui t'avait vu faire ${ }^{14}$. »

Dans les forums, les fils de poèmes, souvent très beaux, les « Hikayate el ghorba " de yabiladi.com, "Des mots, des ombres... des vies" du site sénégalais Sentoo, les poèmes et souvenirs d'enfance en prose de "vie en diaspora » de Netarménie.com comptent parmi les thèmes les plus fréquentés et suscitent des milliers de posts.

Le forum est aussi le lieu de la première construction identitaire ou de sa réactivation selon l'ancienneté et la solidité de la communauté. Plus collaboratif que la zone éditoriale contrôlée par un petit nombre d'auteurs, il reflète les négociations identitaires qui se jouent au sein de la communauté. La question «Selon vous la communauté maghrébine en France est-elle sur le point d'imploser ? ", lancée en novembre 2008 sur le forum d'orientalement.com, conduit à de nombreux débats: s'assimiler ou organiser une communauté maghrébine ? Et d'ailleurs parler de communauté maghrébine a-t-il un sens ? Faut-il confondre Maghreb et Islam ? Comment promouvoir l'image des Maghrébins ? « Ce qui manque à la communauté c'est de l'organisation et de la solidarité ${ }^{15}$ » conclut un internaute.

\footnotetext{
${ }^{14}$ Site www.facebook.com Groupe « Tu sais que tu es un Comorien de France quand... ».

Forum orientalement.com. échanges du 3 novembre 2008 au 2 janvier 2009. http://www.orientalement.com/p1632-selon-vous-la-communaute-maghrebine-en-france-est-elle-surle-point-d-imploser.html (accédé le 1er février 2009).
} 


\section{Claire SCOPSI}

Pour lever ces doutes, la communauté dispose grâce aux innovations du web de moyens de plus en plus sophistiqués pour se convaincre de la réalité de son unité.

Communauté imaginée dont les membres ne se rencontreront jamais mais prennent conscience de leur existence mutuelle et de leur " mêmeté " grâce à des URL et des moteurs de recherche, la diaspora a deux gageures: figurer le homeland et se représenter.

La re-création du homeland passe par l'image et le son. La plupart des sites africains se sont dotées de play-lists musicales au début des années 2000. Les web-radios se sont installées rapidement lorsque la radio traditionnelle était déjà un média développé. C'est le cas de la webradio Malgache Dago Radio Sound, créée en 2004, qui affiche clairement son objectif et sa cible :

« Nous avons voulu créer un lien qui unirait tous les Malgaches du monde entier afin que, même loin de l'lle Rouge, tous les Malgaches puissent retrouver un bout de leur terre et de "leur chez-soi" à travers DRS $\mathrm{RADIO}^{16}$. ॥

Les combinaisons d'effets multimedia sont particulièrement remarquables dans les "sites de village ». Le site du village comoréen de Ntsaoueni ${ }^{17}$ propose depuis 2004 des visites guidées virtuelles sous forme de photos dans un premier temps, puis de vidéo. Les vues de Google Earth sont insérées : «à droite de la photo, vous pourrez reconnaître la maison de Zawad » est-il précisé. Le village de Fornelos del Bollo ${ }^{18}$ (Galice) est littéralement recréé sur le web par ses membres émigrés: les maisons sont photographiées une à une et une webcam est placée au centre du village. Le village kabyle de Taddert-Oufella ${ }^{19}$ est photographié sous toutes les coutures. Les décès, mariages, bacheliers et tournois sportifs sont soigneusement listés. Pour les migrants modernes, le vaet-vient virtuel relaie celui des vacances d'été !

Où sommes-nous ? Combien sommes-nous ? La diaspora se représente en chiffres et en cartes. Beaucoup posent simplement sur un atlas les chiffres officiels des recensements, ou répertorient leurs associations. D'autres construisent cette représentation à partir d'éléments électroniques : le nombre d'inscrits au groupe sur un réseau social, l'origine des participants à un forum. Début 2009, Facebook organise une amicale compétition : le classement des

16 http://www.dagoradiosound.info/but-station-radio-malgache-dago-radio-sound-madagascar.html (accédé le 1er février 2009).

${ }^{17} \mathrm{http}: / /$ htsaoueningome.free.frl (accédé le 1er février 2009).

${ }_{18} \mathrm{http://fornelos.free.fr/indexc.htm} \mathrm{(accédé} \mathrm{le} \mathrm{1er} \mathrm{février} \mathrm{2009).}$

19 http://www.taddertoufella.com/ (accédé le 1er février 2009). 
Les sites web diasporiques : un nouveau genre médiatique?

groupes nationaux les plus représentés sur le réseau social ${ }^{20}$. La communauté libanaise, dont les 55000 membres sont localisés dans le monde entier, se passionne pour le concours et se classe troisième. Dans le classement par continent, l'Afrique devance de loin l'Amérique du Nord et l'Europe. Une façon d'inverser la fracture numérique?

\section{Conclusion : un nouvel âge des diasporas ?}

Le web de migrants est-il un nouveau genre ? Partiellement oui, car tout en s'inscrivant dans une tradition médiatique il profite des innovations des nouveaux médias pour développer de nouveaux contenus et accélérer les échanges. Les thèmes demeurent pourtant étrangement semblables et résistent au temps comme ils résistent aux déclinaisons culturelles. Ce que nous confirme pour l'instant la visite de ces sites, c'est que les thèmes diasporiques sont des invariants. Quelles que soient leurs caractéristiques techniques, pages statiques, forums, blogs ou réseaux sociaux affichent les mêmes débats, les mêmes poèmes, les mêmes nostalgies. D'ailleurs, bien avant les blogs et les réseaux sociaux, les sites statiques de la fin des années 1990 parvenaient à leur façon à être collaboratifs car l'auteur relayait les photos et les commentaires de ses visiteurs et par sa médiation le site s'enrichissait collaborativement. Les logiciels de gestion de contenu n'ont donc pas fondamentalement modifié les pratiques mais ils ont profondément bouleversé la temporalité des échanges.

La communauté prend conscience de ses localisations et de ses points communs de plus en plus rapidement : il suffit d'inscrire le nom d'un pays, d'un village dans le formulaire de Google pour prendre pied dans la diaspora. Ce phénomène mérite une attention particulière car ni le téléphone, ni la télévision satellitaire n'avaient jusqu'à présent permis ce type d'échange instantané à la fois communautaire et individualisé. Cela explique pourquoi de plus en plus de communautés se désignent instantanément comme diasporiques alors que les définitions savantes exigent la durée au-delà de plusieurs générations pour reconnaître à une communauté le nom de diaspora. Le nouveau temps médiatique va-t-il accélérer le processus diasporique ? Si c'est le cas ce sera la fin de la diaspora en tant que communauté d'exception. Si des communautés toujours plus nombreuses parviennent grâce au web à mobiliser leurs forces éparses, c'est tout le jeu des influences ethniques sur les nations qui se trouvera modifié et composer avec les diversités deviendra pour les gouvernements un exercice majeur.

${ }^{20}$ The Great Facebook Race - The World http://www.facebook.com/group.php?gid=2733690506 (accédé le 1er février 2009). 
Claire SCOPSI

\section{Références bibliographiques}

(Les sites web indiqués ont été consultés en février 2009).

ANDERSON B., 1996, L'Imaginaire national : réflexions sur l'origine et l'essor du nationalisme, Paris, La Découverte.

ANTEBY-YEMINI L. , W. BERTHOMIERE et G. SHEFFER, 2005, Les diasporas, 2000 ans d'histoire, Rennes, Presses universitaires de Rennes.

BARRIER N.G., 2006 , « Trauma and memory within the Sikh diaspora : Internet dialogue », Sikh Formations, vol. 2, issue 1, pp.33-56.

BRUNEAU M., 2004, Diasporas et espaces transnationaux, Paris, Anthropos.

COHEN R., 1999, «Diasporas and the nation-state: from victims to challengers ", dans S. VERTOVEC, R. COHEN, Migration, diasporas and transnationalism, Cheltenham, Edward Elgar, pp.266-279.

DAYAN D.L. et KATZ E., 1996, La télévision cérémonielle, Paris, Presses universitaires de France (La politique éclatée).

DIMINESCU D., 2002, «L'usage du téléphone portable par les migrants en situation précaire », Hommes et Migrations, $\mathrm{n}^{\circ} 1240$, pp.66-81, [en ligne] <URL : http://www.ticm.msh-paris.fr/spip.php?article26>.

DIMINESCU D., 2005, «Les documents numériques : méthodologie d'archivage », Migrance, $\mathrm{n}^{\circ} 23$.

DIMINESCU D., 2005, «Le migrant connecté. Pour un manifeste épistémologique », Migrations/Société, vol. 17, n¹02, pp.275-292.

DUFOIX S., 2003, Les diasporas, Paris, Presses universitaires de France, Coll. "Que sais-je?».

GONIN P., 2001, «Migrations développement: les utopies des années 90 », dans R. BLION et I. RIGONI (dir), D'un voyage à l'autre. Des voix de l'immigration pour un développement pluriel, Paris, Éditions Karthala, pp.2548.

IGNACIO E. N., 2006, «E-Spacing boundaries: Bridging cyberspace and diaspora studies through nethnography », dans D. SILVER, A. MASSANARI et S. JONES (dir), Critical Cyber-Culture Studies, New York, New York University Press, pp.181-193. 
MA MUNG E. , 2002, "La désignation des diasporas sur internet », Hommes et Migrations, $\mathrm{n}^{\circ} 1240$, pp.19-27.

MARTHOZ J.-P., 2001, «Médias et "va-et-vient" communicationnel des diasporas », dans R. BLION et I. RIGONI (dir), D'un voyage à l'autre. Des voix de l'immigration pour un développement pluriel, Paris, Éditions Karthala, pp. 189-205.

MEDAM A., 1993, "Diaspora/Diasporas. Archétype et typologie ", Revue européenne des migrations internationales, vol. 9 , n¹ , pp.59-66.

ROLLAN F., 2005, "Une sélection de 90 sites web sur les diasporas », Cybergeo. Espace, société, territoire, article 323, [mis en ligne le 7 novembre 2005, modifié le 22 juin 2007]. <URL : http://www.cybergeo.eu/index3046.html>.

SAYAD A. , 1985, «Du message oral au message sur cassette: la communication avec l'absent », Actes de la recherche en sciences sociales, $\mathrm{n}^{\circ} 59$, septembre, pp.61-73.

SAYAD A., 1991, L'Immigration ou le paradoxe de l'altérité, Bruxelles, De Boeck.

SCHNAPPER D., 2005, " De l'État nation au monde transnational : du sens et de l'utilité du concept de diaspora ", dans L. ANTEBY-YEMINI, W. BETHOMIĖRE et G. SCHEFFER (dir), Les diasporas : 2000 ans d'histoire, Rennes, Presses Universitaires de Rennes.

SCHEFFER G., 1993, "Ethnic diasporas : a threat to their hosts ? " dans M. WEINER (dir), International migration and security, Boulder, Westview Press, pp.263-285.

SCOPSI C., 2004, « Représentation des TIC et multiterritorialité : le cas des télé et cyberboutiques de Château rouge, à Paris », dans A. CHÉNEAULOQUAY (dir), Mondialisation et technologies de la communication en Afrique, Paris-Pessac, Karthala-MSHA.

SCOPSI C., 2008, "Les boutiques de commmunication à Château rouge (Paris) : une contribution privée à la réduction de la fracture numérique », dans Actes du 11ème Colloque international sur le document numérique : CIDE'08, 28-31 octobre 2008, Rouen [en ligne]. <URL : http://www.tic.ird.fr/article.php3?id article=289. $>$

\section{Sites observés}

Abidjan.US Portail http://www.abidjan.us 
Claire SCOPSI

africast.com http://www.africast.com/

Agadez Niger - Diaspora, Touaregs, Artisanat, Voyage http://www.agadezniger.com/

Algérie - Le site de Djamila

http://users.antrasite.be/ppoisse/Documents/algerie.htm

Aujourd'hui Le Maroc - Éloge de la diaspora marocaine

http://www.aujourdhui.ma/culture-details8471.html

Bienvenue au village de Taddert-Oufella O-T-O

http://www.taddertoufella.com/tof vila6.php

Bladi.net (Archives du forum )- fil de discussion « Maroc - France »

http://www.bladi.net/forum/archive/f-19-p-6.html

Diascom.org Le Portail Web de la Coordination des associations de la Diaspora Comorienne http://diascom.org/

Diaspora Grecque http://diaspora-grecque.com/

FarsiNet : A Persian Iranian Farsi Speaking People Global eCommunity http://www.farsinet.com/pwo/diaspora.html

FORIM - Forum des organisations de solidarité internationale issues des migrations http://www.forim.net/fr/index.php

Fornelos del Bollo (Gallice) < http://fornelos.free.fr/indexc.htm>

Irish Emigrant - News and jobs for the global Irish community

http://www.emigrant.ie

Maroc - MRE http://www.mre.ma

Mathematicians of the African Diaspora http://www.math.buffalo.edu/mad

Mondeberbere.com - Amadalamazigh.com - Berberworld.com

http://www.mondeberbere.com

Netarmenie http://www.netarmenie.com/pointsdevue/diaspora/diaspo.php

Nouchi.com La culture africaine avec le sourire (Côte d'Ivoire)

http://www.nouchi.com/

Ntsaoueningomé (Comores) http://ntsaoueningome.free.fr/

Orientalement.com http://www.orientalement.com

Oumma.com http://oumma.com/

Portail Sentoo - Le nouveau regard internet sur le monde (Sénégal)

http://www.forum.sentoo.sn/"

tic\&société - 3 (1-2), 2009 
Les sites web diasporiques : un nouveau genre médiatique?

Soninkara.com <http://www.soninkara.com/>

The Scottish Diaspora Forum http://scottishdiasporaforum.org/scots

Tu sais que tu es un Comorien de France quand... Facebook

http://www.facebook.com/group.php?gid=22607002839

vietkieu La diaspora vietnamienne

http://www.livinginvietnam.com/Fr/vietnam/vietkieu.htm

www.agnamcivol.org le site de l'Association de Développement et de Solidarité du village d'Agnam Civol (Sénégal) en

Francehttp://tbtconception.com/agnamcivol/news.php?lng=fr

Yabiladi.com - Le Maroc à portée de clic ! http://www.yabiladi.com/ 